\title{
Orengedoku-to augmentation in cases showing partial response to yokukan-san treatment: a case report and literature review of the evidence for use of these Kampo herbal formulae
}

\author{
This article was published in the following Dove Press journal: \\ Neuropsychiatric Disease and Treatment \\ 17 January 2013 \\ Number of times this article has been viewed
}

\author{
Hideki Okamoto' \\ Atsushi Chino' \\ Yoshiro Hirasaki' \\ Keigo Ueda' \\ Masaomi lyo ${ }^{2}$ \\ Takao Namiki \\ 'Department of Japanese-Oriental \\ (Kampo) Medicine, Chiba University \\ Graduate School of Medicine, Chiba \\ City, Japan; ${ }^{2}$ Department of Psychiatry, \\ Chiba University Graduate School of \\ Medicine, Chiba City, Japan
}

\begin{abstract}
Background: Yokukan-san, a Japanese traditional herbal (Kampo) prescription, has recently gathered increasing attention due to accumulating reports showing its remarkable efficacy in treating a wide variety of diseases refractory to conventional medicine as well as the behavioral and psychological symptoms of dementia. As yokukan-san has become broadly integrated with conventional medicine, augmentation therapy with other Kampo prescriptions has become necessary when the yokukan-san has been only partially efficacious. In this paper, we report three cases in which the addition of orengedoku-to, another Kampo formula, to yokukan-san was remarkably effective.
\end{abstract}

Cases: Case 1 was an 85-year-old man with Alzheimer-type dementia who had become aggressive during the past 2 years. Three milligrams of aripiprazole completely suppressed his problematic behaviors but had to be stopped because of extrapyramidal symptoms. In the second case, a 44-year-old man with methamphetamine-induced psychosis had suffered from serious tardive dystonia for 2 years. No conventional approach had improved his tardive dystonia. The third case was a 29-year-old engineer who often failed to resist aggressive impulses and was diagnosed with intermittent explosive disorder. He was prescribed $5 \mathrm{mg}$ of olanzapine, which did not suppress his extraordinary anger and caused somnolence even though the dose was low.

Interventions and outcomes: Yokukan-san was complementarily added to the patients' regular medication and exerted a definitive but partial effect in all cases. The addition of orengedoku-to to yokukan-san exerted the same efficacy as aripiprazole in controlling aggressiveness in Case 1, improved the tardive dystonia by $80 \%$ in Case 2, and was completely effective in controlling the patient's aggressive impulses in Case 3.

Conclusion: Together with empirical evidence demonstrating the effectiveness of both yokukansan and orengedoku-to in reducing irritability, impulsivity, and aggression, these three cases suggest that orengedoku-to augmentation can be an effective option in cases that are partially responsive to yokukan-san treatment.

Keywords: traditional medicine, aggressiveness, tardive dystonia, intermittent explosive disorder

\section{Introduction}

Yokukan-san (also called "yoku-kan-san," "yokukansan," and "yi-gan-san"; Tsumura and Company, Tokyo, Japan), a Japanese traditional herbal (Kampo) prescription, has recently gathered increasing attention in the field of psychiatry due to accumulating reports showing its remarkable efficacy in treating behavioral and psychological 
symptoms of dementia (BPSD), as reviewed in $2010 .{ }^{1}$ In addition, various therapeutic effects of yokukan-san have been reported in the treatment of other symptoms such as those of treatment-resistant schizophrenia, ${ }^{2}$ tardive dyskinesia, ${ }^{3}$ borderline personality disorder, ${ }^{4}$ and Huntington's disease. ${ }^{5}$ Today, such differing uses of yokukan-san are consistent with the uses of yokukan-san described in classic medical books. Yokukan-san has been utilized for more than 400 years, since before the establishment of conventional medicine, based on Kampo-specific indications. Yokukan-san is officially listed in the Japanese Guideline for the Management of Dementia 2010 by the Japanese Society of Neurology (http:// www.neurology-jp.org/guidelinem/nintisyo.html) and will continue to be more broadly integrated with conventional medication in treating various diseases including dementia. As the use of yokukan-san becomes more widespread, augmentation therapy with other Kampo prescriptions will become necessary when the yokukan-san is only partial efficacious. Developing such augmentation therapy can broaden the application of Kampo medicine in a wide variety of diseases refractory to conventional medicine.

Here, we report three cases in which the addition of orengedoku-to (Tsumura and Company), another Kampo formula, to yokukan-san was remarkably effective.

\section{Case I}

An 85-year-old male had been treated with donepezil for 5 years due to Alzheimer's-type dementia. During these 5 years, he had exhibited increasingly aggressive behaviors, directed especially toward his wife, that he had not demonstrated before. He engaged in dangerous behaviors driven by delusions or hallucinations as a result of his hearing murmuring advising him that he was about to be killed.

His behaviors were consistent with the behavioral and psychological symptoms of dementia and treatment with $3 \mathrm{mg}$ of aripiprazole was started. The aripiprazole completely suppressed the aggressive behaviors after 4 weeks but caused extrapyramidal symptoms such as mild goiter, mild steppage gait, and moderate tremor, even at this low dose. Therefore, yokukan-san ( $5 \mathrm{~g} /$ day) was prescribed in substitution for the aripiprazole according to the Japanese Guideline for the Management of Dementia 2010 for the treatment of BPSD, and it worked to suppress the patient's BPSD for the first 16 weeks. Following this, the patient gradually became aggressive again, although the aggression was milder than before. Thus, orengedoku-to ( $5 \mathrm{~g} /$ day) was added to the yokukan-san. The combination therapy of yokukan-san and orengedoku-to, showed an efficacy equal to that of aripiprazole without any adverse effects.

\section{Case 2}

A 44-year-old male had started to use methamphetamine at the age of 20 . When he was 32 years old, he began to exhibit signs of methamphetamine-induced psychotic disorder accompanied by perceptional delusions and auditory hallucinations and started to take antipsychotic medication irregularly. After twice serving prison time, the patient stopped using methamphetamine and began taking $6 \mathrm{mg}$ of risperidone regularly at the age of 42 , which caused severe tardive dystonia affecting his whole body. His tardive dystonia was refractory to conventional medication such as the maximum doses of tizanidine - a centrally acting skeletal muscle relaxant - benzodiazepines, and anticholinergic drugs, as well as other atypical antipsychotics like $10 \mathrm{mg}$ of olanzapine or $30 \mathrm{mg}$ of aripiprazole. When he arrived at our hospital, he was unable to sit still in the waiting room.

In addition, he had been irritable and aggressive toward his mother, who lived with him, which had often resulted in the destruction of property at home. When yokukan-san (7.5 g/day) was added to his conventional medication, his involuntary movements were reduced by $30 \%$ after 2 weeks. Then, when orengedoku-to (7.5 g/day) augmentation was started after 4 weeks, the tardive dystonia was reduced by $80 \%$ after 6 weeks. He was able to sit still on the same bench with other patients in the waiting room for the first time in 2 years and property destruction became a much less common occurrence, although his attitude toward his mother remained abrupt.

\section{Case 3}

A 29-year-old engineer with a doctorate degree often failed to resist aggressive impulses even in front of his employer at work. The degree of aggressiveness resulted in the destruction of property every few months, which he greatly regretted each time. He was diagnosed with intermittent explosive disorder (IED) based on the Diagnostic and Statistical Manual of Mental Disorders 4th Edition ${ }^{6}$ criteria and was prescribed olanzapine $(5 \mathrm{mg})$. Although the olanzapine treatment was partially effective and he was able to resist violent impulses to destroy property, he still felt not only extraordinarily angry at times but also somnolent due to the olanzapine, even though the dose was low.

We added yokukan-san (2.5 g) as a complement to the olanzapine medication up to three times daily as needed. The patient voluntarily chose to take yokukan-san regularly three times per day ( $7.5 \mathrm{~g}$ in total) because he found that it had remarkable efficacy in helping him to resist engaging in impulsive behavior. He decided to stop taking the 
olanzapine and asked permission to take the Kampo medicine alone, hoping that it would be more effective. In response to this request, we added orengedoku-to $(7.5 \mathrm{~g} /$ day $)$ to the yokukan-san, and the combination therapy of yokukan-san and orengedoku-to was completely effective in controlling his aggressive impulses, resulting in a Clinical Global Impressions - Improvement scale score of 1 (very much improved). Thereafter, the patient no longer felt the extreme anger he had experienced in the past.

\section{Discussion}

Both yokukan-san and orengedoku-to are extract granules manufactured as aqueous extracts containing herbs by strictly licensed pharmaceutical companies in Japan. The Japanese Ministry of Health, Labor, and Welfare has approved 148 orally administered Kampo formulae, composed of herbal extract granules, for clinical use in the same way as conventional medicines are used. Therapy based on Kampo medicine was the main medical treatment in Japan until Western medicine was introduced from Europe several hundred years ago. Recently, Kampo medicine has begun to receive increasing renewed attention because it provides a valid alternative approach to treating symptoms refractory to conventional medicine. ${ }^{7-9}$

Yokukan-san, which is comprised of seven herbs (Angelicae Radix, Atractylodis Lanceae Rhizoma, Bupleuri Radix, Poria, Glycyrrhizae Radix, Cnidii Rhizoma, and Uncariae Uncis Cum Ramlus) (Table 1), was listed in a Chinese medical book in $1555 \mathrm{AD}$ for the first time and thereafter came to Japan. The name "yokukan-san" originally meant "medicine suppressing the liver." The original Chinese description states that yokukan-san treats spasms, feverishness, clenching, terrified/ simmering mental state, or intolerance to heat and cold caused by pseudo-hyperfunction of the liver and expectoration of sputum, abdominal fullness, loss of appetite, and sleep disorders caused by dysfunction of the spleen, which has a restraining relationship with the liver and is consequently damaged by the liver's pseudo-hyperfunction.

Table I Components of yokukan-san

\begin{tabular}{ll}
\hline Constituent herbs & Weight (g) \\
\hline Angelicae Radix (当帰) & 3.0 \\
Atractylodis Lanceae Rhizoma (蒼术) & 4.0 \\
Bupleuri Radix (柴胡) & 2.0 \\
Poria (获苓) & 4.0 \\
Glycyrrhizae Radix (甘草) & 1.5 \\
Cnidii Rhizoma (川芦) & 3.0 \\
Uncariae Uncis Cum Ramlus (釣藤鈎) & 3.0 \\
\hline
\end{tabular}

According to the Five Phases theory, the liver is considered to be the organ that stabilizes mental activities and ameliorates involuntary muscle movements, and thus the liver often produces anger and/or involuntary muscle movement when its regular functioning is impaired. ${ }^{8,9}$ Many famous Kampo medicine doctors agreed with the use of yokukan-san for these indications. For example, Dotaku Meguro (1739-1798 AD) wrote that yokukan-san must have some efficacy if the patient feels anger, and Sohaku Asada (1815-1894 AD) wrote that yokukan-san can be used to treat a patient who has muscle spasms and anger, and all of his symptoms are likely to be caused by pseudo-hyperfunction of the liver. Based on these indications, yokukan-san has been used in Japan for more than 400 years as a treatment for insomnia, irritability, impulsivity, aggression, blepharospasm, tremor or fasciculation of the facial muscles, convulsions, and similar symptoms. ${ }^{7-9}$ It is not surprising that yokukan-san has lately been reported to have ameliorating effects in such a wide variety of diseases because it has long been considered in China and Japan to exert these, irrespective of conventional diagnosis, at any time patients exhibit aggressiveness, impulsive behaviors, and/or involuntary movements.

Orengedoku-to, which consists of four herbs (Coptidis Rhizoma, Scutellariae Radix, Phellodendri Cortex, and Gardeniae Fructus) (Table 2), was described in a Chinese medical book of $752 \mathrm{AD}$ as being able to treat patients suffering from excessive body heat, mental conflict, confusion, insomnia, thirst, and nausea. In ancient times, orengedoku-to was used by soldiers before battle, both to reduce their anger so they would not become confused as well as to prevent excessive bleeding. ${ }^{7}$ As might be expected from its history, this compound has come to be used for the treatment of ailments involving bleeding (eg, nosebleeds, hemorrhoids), hypertension, irritation, and face flushing, as well as for inflammatory diseases such as gastritis. ${ }^{7}$ In fact, it has been proven in animal models that orengedoku-to has protective effects against stress-induced gastric ulcers and gastritis. ${ }^{10}$ Thus, orengedoku-to has a "cooling" effect on inflammatory symptoms, whether they have mental or physical causes, and is often used to treat irritability, impulsivity, aggression,

Table 2 Components of orengedoku-to

\begin{tabular}{ll}
\hline Constituent herbs & Weight (g) \\
\hline Coptidis Rhizoma (黄連) & 2.0 \\
Scutellariae Radix (黄芩) & 3.0 \\
Phellodendri Cortex (黄柏) & 1.5 \\
Gardeniae Fructus (山桅子) & 2.0 \\
\hline
\end{tabular}


pruritus, dermatitis, and other diseases, irrespective of conventional diagnosis, in which patients are considered to have excessive heat in their bodies and/or hearts. In most cases in which orengedoku-to is effective, patients have a tendency to have a reddish face and feelings of being increasingly hot. $^{7-9}$

Thus, based on past use, both yokukan-san and orengedoku-to are thought to be effective for treating irritability, impulsivity, and aggression. Nevertheless, today yokukan-san tends to be chosen first due to its reputation for effectively treating many psychiatric symptoms. This is simply because orengedoku-to is still not known among conventional medical doctors due to the lack of evidence for its effectiveness. As already suggested, orengedoku-to by itself is more likely to be effective in cases in which patients suffer not only from irritability, impulsivity, and aggression, but also a reddish face and feelings of being increasingly hot. $^{7-9}$ However, even in cases in which patients do not have a reddish face or feelings of being increasingly hot, orengedoku-to should be considered as an alternative treatment for irritability, impulsivity, and aggression, given that the patients with those symptoms have excessive heat not in their bodies but in their hearts. Therefore, in patients who do not feel physically cold, the addition of orengedoku-to's cooling effect to yokukan-san can be an effective option in cases that are partially responsive to yokukan-san treatment. In fact, Coptis rhizome and Gardenia fruit, two of the four components of orengedoku-to, are often added to the decoction medicine of yokukan-san to augment its efficacy, as described in classic Japanese medical books. In particular, Coptis rhizome, the common name of which is "oren" in Japanese and which is the main component of orengedoku-to, is frequently added to yokukan-san. Therefore, orengedoku-to augmentation in cases that are partially responsive to yokukan-san is also very reasonable when granule prescriptions but not the decoction medicines are available.

Three open-label studies support the notion that the use of yokukan-san is beneficial in BPSD and in treating behaviors of Alzheimer's-type dementia, ${ }^{11-13}$ and our results suggest that the addition of orengedoku-to to yokukansan may be more effective in treating Alzheimer's-type dementia than yokukan-san alone, especially in cases showing aggression. Further, a recent report has shown that orengedoku-to may have therapeutic potential in patients with Alzheimer's disease by inhibiting indoleamine 2,3-dioxygenase activity. ${ }^{14}$ In addition, the combination therapy involving yokukan-san and orengedoku-to is likely to be effective, irrespective of conventional diagnosis, under conditions suitable for both yokukan-san and orengedoku-to as described, because Kampo medicine often shows remarkable effectiveness in cases that, according to the Kampo diagnosis, meet the criteria for choosing each Kampo prescription. ${ }^{7-9}$

\section{Conclusion}

Together with empirical use demonstrating the effectiveness of both yokukan-san and orengedoku-to in reducing irritability, impulsivity, and aggression, these three cases suggest that orengedoku-to augmentation can be an effective option in cases that are partially responsive to yokukan-san treatment. The therapeutic mechanisms of both medicines remain unclear, and further studies are needed to increase their usefulness in clinical practice.

\section{Disclosure}

No commercial party having a direct financial interest in the results of the research supporting this article has or will confer a benefit upon the authors or upon any organization with which the authors are associated.

\section{References}

1. de Caires S, Steenkamp V. Use of Yokukansan (TJ-54) in the treatment of neurological disorders: a review. Phytother Res. 2010;24(9): $1265-1270$

2. Miyaoka T, Furuya M, Yasuda H, et al. Yi-gan san as adjunctive therapy for treatment-resistant schizophrenia: an open-label study. Clin Neuropharmacol. 2009;32(1):6-9.

3. Miyaoka T, Furuya M, Yasuda H, et al. Yi-gan san for the treatment of neuroleptic-induced tardive dyskinesia: an open-label study. Prog Neuropsychopharmacol Biol Psychiatry. 2008;32(3): 761-764.

4. Miyaoka T, Furuya M, Yasuda H, Hayashia M, Inagaki T, Horiguchi J. Yi-gan san for the treatment of borderline personality disorder: an openlabel study. Prog Neuropsychopharmacol Biol Psychiatry. 2008;32(1): $150-154$.

5. Satoh T, Takahashi T, Iwasaki K, et al. Traditional Chinese medicine on four patients with Huntington's disease. Mov Disord. 2009;24(3): 453-455.

6. American Psychiatric Association. Diagnostic and Statistical Manual of Mental Disorders: DSM-IV; Fourth Edition. Arlington, VA: American Psychiatric Association; 1994.

7. Okamoto H. Reconsideration of Japanese traditional herbal medicine: new field of research and clinical medicine. Mini Rev Med Chem. 2006;6(5):543-547.

8. The Japan Society for Oriental Medicine. Introduction to Kampo: Japanese Traditional Medicine. Tokyo: Elsevier Japan; 2005.

9. Terasawa K. Kampo. Tokyo: Standard McIntyre; 1993.

10. Ohta Y, Kobayashi T, Nishida K, Sasaki E, Ishiguro I. Preventive effect of Oren-gedoku-to (Huanglian-Jie-Du-Tang) extract on the development of stress-induced acute gastric mucosal lesions in rats. J Ethnopharmacol. 1999;67(3):377-384. 
11. Monji A, Takita M, Samejima T, et al. Effect of yokukansan on the behavioral and psychological symptoms of dementia in elderly patients with Alzheimer's disease. Prog Neuropsychopharmacol Biol Psychiatry. 2009;33(2):308-311.

12. Hayashi Y, Ishida Y, Inoue T, et al. Treatment of behavioral and psychological symptoms of Alzheimer-type dementia with Yokukansan in clinical practice. Prog Neuropsychopharmacol Biol Psychiatry. 2010;34(3):541-545.
13. Okahara K, Ishida Y, Hayashi Y, et al. Effects of Yokukansan on behavioral and psychological symptoms of dementia in regular treatment for Alzheimer's disease. Prog Neuropsychopharmacol Biol Psychiatry. 2010;34(3):532-536.

14. Yu CJ, Zheng MF, Kuang CX, Huang WD, Yang Q. Oren-gedoku-to and its constituents with therapeutic potential in Alzheimer's disease inhibit indoleamine 2, 3-dioxygenase activity in vitro. $J$ Alzheimers Dis. 2010;22(1):257-266.

Neuropsychiatric Disease and Treatment

\section{Publish your work in this journal}

Neuropsychiatric Disease and Treatment is an international, peerreviewed journal of clinical therapeutics and pharmacology focusing on concise rapid reporting of clinical or pre-clinical studies on a range of neuropsychiatric and neurological disorders. This journa is indexed on PubMed Central, the 'PsycINFO' database and CAS
The manuscript management system is completely online and includes a very quick and fair peer-review system, which is all easy to use. Visit http://www.dovepress.com/testimonials.php to read real quotes from published authors.

Submit your manuscript here: http://www.dovepress.com/neuropsychiatric-disease-and-treatment-journal 